\title{
Simultaneous mechanical-scan-free confocal microscopy and laser microsurgery
}

\author{
Kevin K. Tsia, Keisuke Goda,* Dale Capewell, and Bahram Jalali \\ Electrical Engineering Department, University of California, Los Angeles, California 90095, USA \\ *Corresponding author: goda@ee.ucla.edu
}

Received February 5, 2009; revised May 28, 2009; accepted June 2, 2009; posted June 10, 2009 (Doc. ID 107209); published July 7, 2009

\begin{abstract}
We demonstrate an endoscope-compatible single-fiber-based device that performs simultaneous confocal microscopy and high-precision laser microsurgery. The method is based on mapping of two-dimensional sample coordinates onto the optical spectrum and allows us to perform two-dimensional imaging and microsurgery without any mechanical movement of the probe or the sample. The technology holds promise for creating highly miniaturized endoscopes for applications such as brain tumor, pediatric, and endovascular surgeries where high-precision, small, and flexible probes are required. (C) 2009 Optical Society of America OCIS codes: $170.2150,180.1790$.
\end{abstract}

Endoscopic tools, such as those employed in colonoscopic, arthroscopic, and thoracoscopic surgeries, have proven to be highly effective for minimally invasive procedures as they reduce the chance of complications, recovery time, and requirements for anesthesia [1]. Endoscopes use a CCD or a fiber bundle for image acquisition and, in some cases, combine it with a separate surgical instrument, such as a scalpel or forceps to provide concurrent visual inspection and in vivo surgery. To be minimally invasive, endoscopes must have a very small diameter and be highly flexible. In CCD-based probes, the size of the chip placed at the distal tip limits the minimum diameter to about a few millimeters, and their electrical cables limit their flexibility. The fiber bundle technology is subject to the fundamental limitation between the number of pixels and probe flexibility, because a high pixel count requires a large number of fibers resulting in mechanical rigidity $[2,3]$.

A highly miniature probe capable of simultaneous in vivo imaging and surgery is of paramount value, in particular, for applications that require the ultimate probe miniaturization, such as brain tumor, pediatric, and endovascular surgeries. This has fueled the development of miniaturized single-fiber-based endoscopes for the past several years [3-6]. While the prior techniques have successfully shown imaging capability with single-fiber probes, their need for mechanical scanning to acquire multidimensional images leads to a twofold limitation in endoscopy. First, fluctuations in mechanical scanning introduce image noise and artifacts [3-6]. This problem is well documented for mechanically scanned ultrasound probes [7]. Similar limitations also exist in endoscopic probes with microelectromechanical system (MEMS) scanners [5,6]. Second, MEMS scanners in miniaturized endoscopic probes inevitably require large probe size up to $\sim 1 \mathrm{~cm}$, limiting their clinical utility.

In this Letter, we propose and demonstrate a new (to our knowledge) single-fiber-based imaging method that eliminates the need for mechanical scanning. It also features simultaneous imaging and microsurgery using the same single fiber. We refer it to as spectral-shower encoded confocal microscopy and microsurgery (SECOMM). The central part of SECOMM is a 2D spatial disperser that transforms incident broadband light into a beam in a $2 \mathrm{D}$ spatial spectral pattern resembling a spectral shower and hence creates a one-to-one mapping between $2 \mathrm{D}$ spatial coordinates of a sample and the optical wavelengths. The 2D spatial information of the sample is then encoded into the spectrum of the backreflected spectral shower, allowing $2 \mathrm{D}$ images of the sample to be transmitted through a single-mode fiber. The imaging system is essentially a confocal microscope as the aperture of the fiber that captures the reflection from the sample rejects the scattered light from outof-focus axial planes.

SECOMM is schematically shown in Fig. 1(a). An incoherent broadband light source (an amplified spontaneous emission source with a center wavelength of $\lambda_{o}=1543 \mathrm{~nm}$ and bandwidth of $\Delta \lambda_{\mathrm{ss}}$ $=17 \mathrm{~nm})$ delivers the light $(\sim 2 \mathrm{~mW})$ via a singlemode fiber, a 2D spatial disperser, and an objective lens (a focal length of $f=4.5 \mathrm{~mm}$ ) to the sample. As shown in Fig. 1(b), the 2D spatial disperser consists

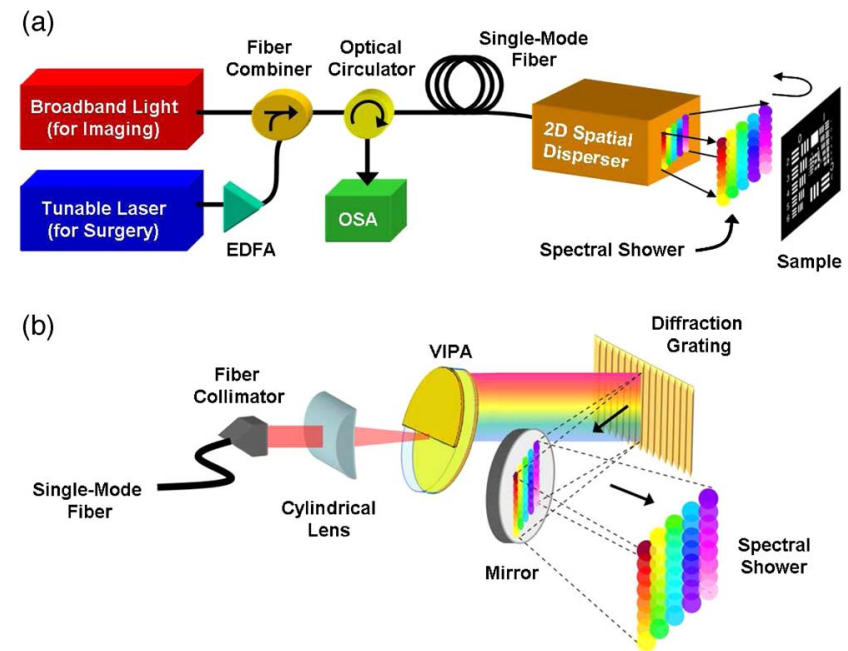

Fig. 1. (Color online) (a) Schematic of the SECOMM. OSA, optical spectrum analyzer; EDFA, erbium-doped fiber amplifier. (b) 2D spatial disperser that consists of a VIPA and a diffraction grating. 
of a pair of spatial dispersers, a diffraction grating (a groove density of $\Lambda=1200$ lines $/ \mathrm{mm}$ ), and a virtually imaged phased array (VIPA) (a thickness of $1.5 \mathrm{~mm}$ ) that is essentially a tilted Fabry-Pérot cavity [8]. Such a grating-VIPA arrangement has previously been used for wavelength demultiplexing in telecommunication applications [9] and for spectroscopy [10]. In contrast, here we employ this $2 \mathrm{D}$ spatial disperser for the purpose of imaging and microsurgery. A collimated broadband beam $(\sim 1 \mathrm{~mm})$ is focused by a cylindrical lens (a focal length of $200 \mathrm{~mm}$ ) onto the front side of the VIPA (a tilted angle of $\theta_{\mathrm{VIPA}}=\sim 2.5^{\circ}$ and a refractive index of $n=1.48$ ) through the uncoated window area. The reflectivity of the coated area on the front and back surface are $99.9 \%$ and $95 \%$, respectively. The spectral shower beam has a beam diameter of $\sim 2 \mathrm{~mm}$, which almost fills the back aperture of the objective lens. The image that is now encoded into the spectrum of the backreflected spectral shower is then directed through the same 2D spatial disperser, after which it reenters the singlemode fiber and is routed, via an optical circulator, to an optical spectrum analyzer (OSA) that captures the image-encoded spectrum. The imaging sensitivity can be enhanced by optical amplification of the image-encoded spectrum using optical fiber amplifiers [11]. The image of the sample is digitally reconstructed by folding the $1 \mathrm{D}$ spectral data into a $2 \mathrm{D}$ matrix that represents the image. The sorting step is done based on the knowledge of the free spectral range (FSR) of the VIPA $\left(\Delta \lambda_{\mathrm{FSR}}=0.54 \mathrm{~nm}\right)$, which corresponds to the column length of the image. The image quality is enhanced by Wiener filtering, image interpolation, and contrast equalization. An additional frequency-tunable $\mathrm{cw}$ laser is used for simultaneous mechanical-scan-free laser ablation and imaging, which will be discussed later.

We first imaged a reference target with SECOMM to demonstrate its imaging functionality. Figure 2(a) shows the measured spectrum of the backreflected spectral shower from the target. The modulation that appears in the spectrum reflects the encoded spatial information of the target. The decoded image is shown in Fig. 2(b).

Based on the Fresnel diffraction analysis with paraxial approximation, the horizontal $(\Delta x)$ and vertical $(\Delta y)$ dimensions of the imaging field of view $(\mathrm{FOV}=\Delta x \Delta y)$ are given by $\Delta x \approx 2 f \Lambda \Delta \lambda_{\mathrm{SS}} / \cos \theta_{g}$ and $\Delta y \approx 2 f \Delta \lambda_{\mathrm{FSR}} /\left(\lambda_{0} \tan \theta_{\mathrm{VIPA}}\right)$, where $\theta_{g}$ is the grating angle $\left(\sim 60^{\circ}\right)$. Here the $x$ axis is in the diffraction plane of the diffraction grating, while the $y$ axis is in the diffraction plane of the VIPA. The estimated field of view is $370 \mu \mathrm{m} \times 110 \mu \mathrm{m}$, which is reasonably close to the measured value of $240 \mu \mathrm{m} \times 110 \mu \mathrm{m}$. This discrepancy is due to the fact that the estimation is based on paraxial approximation.

The spatial resolution in the $x$ direction $(\delta x)$ is defined by the spatial separation between adjacent columns of the spectral shower (separated by one FSR of the VIPA) and is hence given by $\delta x \approx 2 f \Lambda \Delta \lambda_{\mathrm{FSR}} /$ $\cos \theta_{g} \approx 11 \mu \mathrm{m}$. This is valid under the conditions that the spatial smears caused by the diffraction limit given by the NA of the objectives lens, the spec-

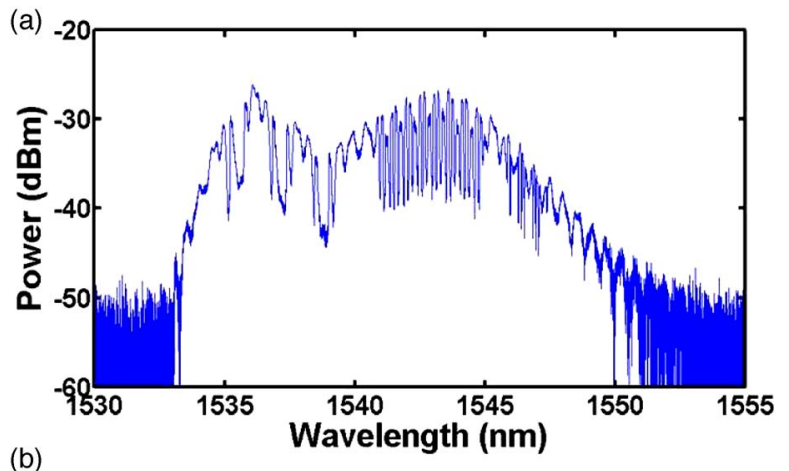

(b)

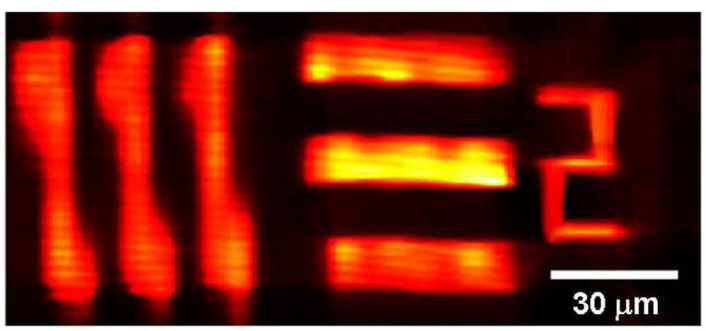

Fig. 2. (Color online) (a) Measured spectrum of the backreflected spectral shower from a reference target. (b) Image of the reference target after image reconstruction.

tral resolution of the diffraction grating, and the quality factor of the VIPA are smaller than the column separation of the spectral shower. On the other hand, the spatial resolution in the $y$ direction ( $\delta y)$ relates to the finite number of phased array elements in the VIPA, which contributes to the interference, analogous to the diffraction grating in which the number of illuminated groove lines defines the spatial resolution. Based on our measurement of the spatial smear of a monochromatic cw laser on top of the spectral shower, we find $\delta y \approx 4 \mu \mathrm{m}$.

Also, the dimension of the pixel size in the $x$ and $y$ directions is given by $\delta X \approx 2 f \Lambda \Delta \lambda_{\mathrm{FSR}} / \cos \theta_{g}$ and $\delta Y \approx 2 f \delta \lambda_{\mathrm{OSA}} /\left(\lambda_{0} \tan \theta_{\mathrm{VIPA}}\right)$, respectively, where $\delta \lambda_{\mathrm{OSA}}$ is the spectral sampling resolution of the spectrometer $\left(\delta \lambda_{\mathrm{OSA}}=0.005 \mathrm{~nm}\right)$. Hence, the number of pixels in the $x$ and $y$ directions is found to be $N_{x}=\Delta x / \delta X$ $=\Delta \lambda_{\mathrm{SS}} / \Delta \lambda_{\mathrm{FSR}}$ and $N_{y}=\Delta y / \delta Y=\Delta \lambda_{\mathrm{FSR}} / \delta \lambda_{\mathrm{OSA}}$, respectively. The total number of pixels is given by $N=N_{x} N_{y}=\Delta \lambda_{\mathrm{ss}} / \delta \lambda_{\mathrm{OSA}}=3400$.

In the second experiment, we showed that the SECOMM technique is capable of simultaneously performing both single-fiber-based imaging and in situ laser ablation. A cw external-cavity frequencytunable diode laser $(1520 \mathrm{~nm}-1570 \mathrm{~nm})$ and an erbium-doped fiber amplifier (EDFA) are added to SECOMM via a fiber combiner to perform laser ablation and to image the process in real time [Fig. 1(a)]. The ablation laser power is $\sim 300 \mathrm{~mW}$. The experiment was performed on a bovine tissue sample. The ablation laser is coupled into the same $2 \mathrm{D}$ disperser that performs the imaging, and hence it follows the same wavelength-to-spatial-coordinate mapping as the spectral shower that is performing the imaging. By tuning the wavelength of the laser, the ablation beam can be directed to any arbitrary position on the tissue without any mechanical movement of the probe or the tissue. Hence high-precision microsur- 
gery can be performed by computer-controlled tuning of the laser wavelength according to a preprogrammed pattern.

Figure 3 shows the images of the tissue captured by SECOMM before and after the laser ablation process, which was completed within $10 \mathrm{~s}$. The random variations appearing in the figure represent the rough surface morphology of the sample. By tuning the wavelength of the ablation laser, an L-shaped pattern within an area of $40 \mu \mathrm{m} \times 40 \mu \mathrm{m}$ on the tissue was ablated. This proof-of-concept experiment illustrates the capability of this instrument to perform in situ high-precision laser microsurgery and simultaneously monitor the process with the same singlefiber probe. This capability bodes well for microsurgery applications that require higher precision than what is achievable with manual manipulation of the surgical probe.

It should be noted that the present image quality is not the inherent limitation of this technique, because the number of pixels can significantly be increased by using an optical source with larger bandwidth or a spectrometer with higher spectral resolution. For instance, the number of pixels can be increased up to $\sim 100,000$ using an optical source with $\sim 200 \mathrm{~nm}$ bandwidth centered around $\sim 800 \mathrm{~nm}$ (e.g., a Ti:sapphire laser) and a 2D spatial disperser-based spectrometer with $\sim 1 \mathrm{GHz}$ spectral resolution $[10,12]$. In addition, the spatial resolution can be improved in the $x$ direction by using a diffraction grating with smaller dispersion and/or a VIPA with smaller FSR. The spatial resolution in the $y$ direction can be improved by using a VIPA with smaller tilt angle to increase the number of virtual phase elements. By careful design of the orientation and position of the VIPA, diffraction grating, and gradient-index lens using ZEMAX, we found that it is feasible to miniaturize SECOMM into a submillimeter-diameter probe

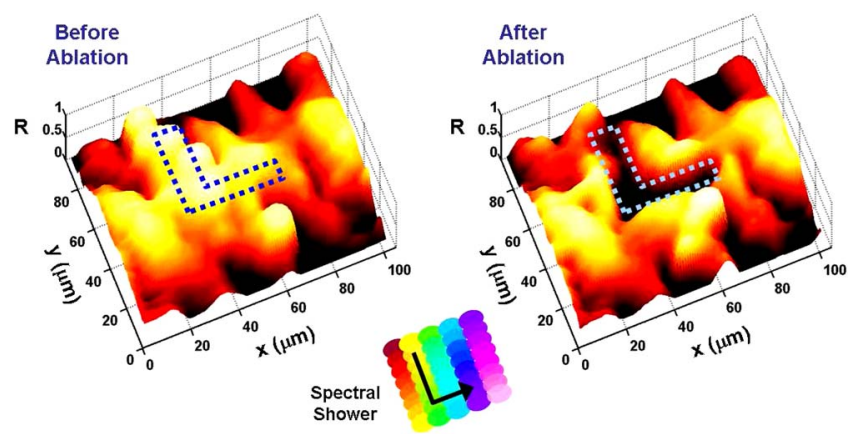

Fig. 3. (Color online) SECOMM images captured before (left) and after (right) laser ablation on a bovine tissue sample. The L pattern (dotted area) is carved out of the tissue by tuning the wavelength of the $\mathrm{cw}$ laser in the manner shown in the inset (see the arrow). The $z$ axis represents the normalized reflectivity of the sample. with spatial resolution of $1.4 \mu \mathrm{m} \times 2 \mu \mathrm{m}$, with a field of view of $280 \mu \mathrm{m} \times 70 \mu \mathrm{m}$. The frame rate in this demonstration was limited to $1 \mathrm{~Hz}$ owing to the low scan rate of our OSA. However, the use of a CCDbased spectrometer can increase the frame rate up to the video rate or higher. Hence, while these limitations can be mitigated by the technology available today (i.e., broadband light sources and miniaturized relay optics), this demonstration represents a step toward realizing the miniaturized optical-fiber endoscope without the need of mechanical scanning for both imaging and laser microsurgery.

In summary, we have proposed and demonstrated a new medical device technology that is capable of performing $2 \mathrm{D}$ imaging and microsurgery with a single optical fiber and without any mechanical steering - a feature that enables miniaturized and flexible endoscopic probes for high-speed, highprecision, and high-throughput microsurgery. For example, it is expected to be an effective method for removing tumors and treating movement disorders such as Parkinson's disease in the deep brain. Finally, we note that the present imaging technique can also be further extended to $3 \mathrm{D}$ volumetric imaging by employing an interferometric configuration, which enables the acquisition of the depth information of the sample.

This work was supported by the Defense Advanced Research Projects Agency (DARPA). We thank D. R. Solli and T. J. Nakamura at UCLA for valuable discussions.

\section{References}

1. N. J. Soper, Mastery of Endoscopic and Laparoscopic Surgery (Lippincott Williams \& Wilkins, 2008).

2. F. Gmitro and D. Aziz, Opt. Lett. 18, 565 (1993).

3. B. A. Flusberg, A. Nimeerjahn, E. D. Cocker, E. A. Mukamel, R. P. J. Barretto, T. H. Ko, L. D. Burn, J. C. Jung, and M. J. Schnitzer, Nat. Methods 5, 935 (2008).

4. D. Yelin, I. Rizvi, W. M. White, J. T. Motz, T. Hasan, B. E. Bouma, and G. J. Tearney, Nature 443, 765 (2006).

5. Y. Pan, H. Xie, and G. K. Fedder, Opt. Lett. 26, 1966 (2001).

6. C. L. Hoy, N. J. Durr, P. Chen, W. Piyawattanametha, H. Ra, O. Solgaard, and A. Ben-Yakar, Opt. Express 16, 9996 (2008).

7. C. Rumack, S. Wilson, J. W. Charboneau, and J.-A. Johnson, Diagnostic Ultrasound (Mosby, 2004).

8. M. Shirasaki, Opt. Lett. 21, 366 (1996).

9. S. Xiao and A. M. Weiner, Opt. Express 12, 2895 (2004).

10. S. A. Diddams, L. Hollberg, and V. Mbele, Nature 445, 627 (2007).

11. K. Goda, K. K. Tsia, and B. Jalali, Nature 458, 1145 (2009).

12. M. J. Thorpe, D. Balslev-Clausen, M. S. Kirchner, and J. Ye, Opt. Express 16, 2387 (2008). 